livraisons

d'Histoire

de l'Architecture

\section{Livraisons de l'histoire de l'architecture}

16 | 2008

Grands chantiers et matériaux

\title{
L'approvisionnement du chantier de l'arc de triomphe du Carrousel : construction et restauration (1806-1933)
}

The supplying of the building site of the Carrousel triumphal arc: construction and restoration (1806-1943)

Die Materialbeschaffung der Baustelle des Arc de triomphe du Carrousel in

Paris : Bau und Restaurierung (1806-1943)

\section{Guillaume Fonkenell}

\section{OpenEdition}

Journals

Édition électronique

URL : http://journals.openedition.org/lha/184

DOI : 10.4000/lha.184

ISSN : 1960-5994

Éditeur

Association Livraisons d'histoire de l'architecture - LHA

Édition imprimée

Date de publication : 10 décembre 2008

Pagination : 71-86

ISSN : 1627-4970

Référence électronique

Guillaume Fonkenell, « L'approvisionnement du chantier de l'arc de triomphe du Carrousel :

construction et restauration (1806-1933)», Livraisons de l'histoire de l'architecture [En ligne], 16 | 2008,

mis en ligne le 10 décembre 2009, consulté le 10 décembre 2020. URL : http://

journals.openedition.org//ha/184 ; DOI : https://doi.org/10.4000//ha.184

Ce document a été généré automatiquement le 10 décembre 2020.

Tous droits réservés à l'Association LHA 


\section{L'approvisionnement du chantier de l'arc de triomphe du Carrousel : construction et restauration (1806-1933)}

The supplying of the building site of the Carrousel triumphal arc: construction and restoration (1806-1943)

Die Materialbeschaffung der Baustelle des Arc de triomphe du Carrousel in Paris : Bau und Restaurierung (1806-1943)

\section{Guillaume Fonkenell}

1 Actuelle porte monumentale ouvrant sur les jardins des Tuileries et le grand axe ouest de Paris, l'arc de triomphe du Carrousel (ill. 1) fut construit avec une intention bien différente : servir d'entrée d'apparat au palais des Tuileries, siège du pouvoir exécutif français durant la plus grande partie du XIX siècle jusqu'à sa destruction en 1871. 


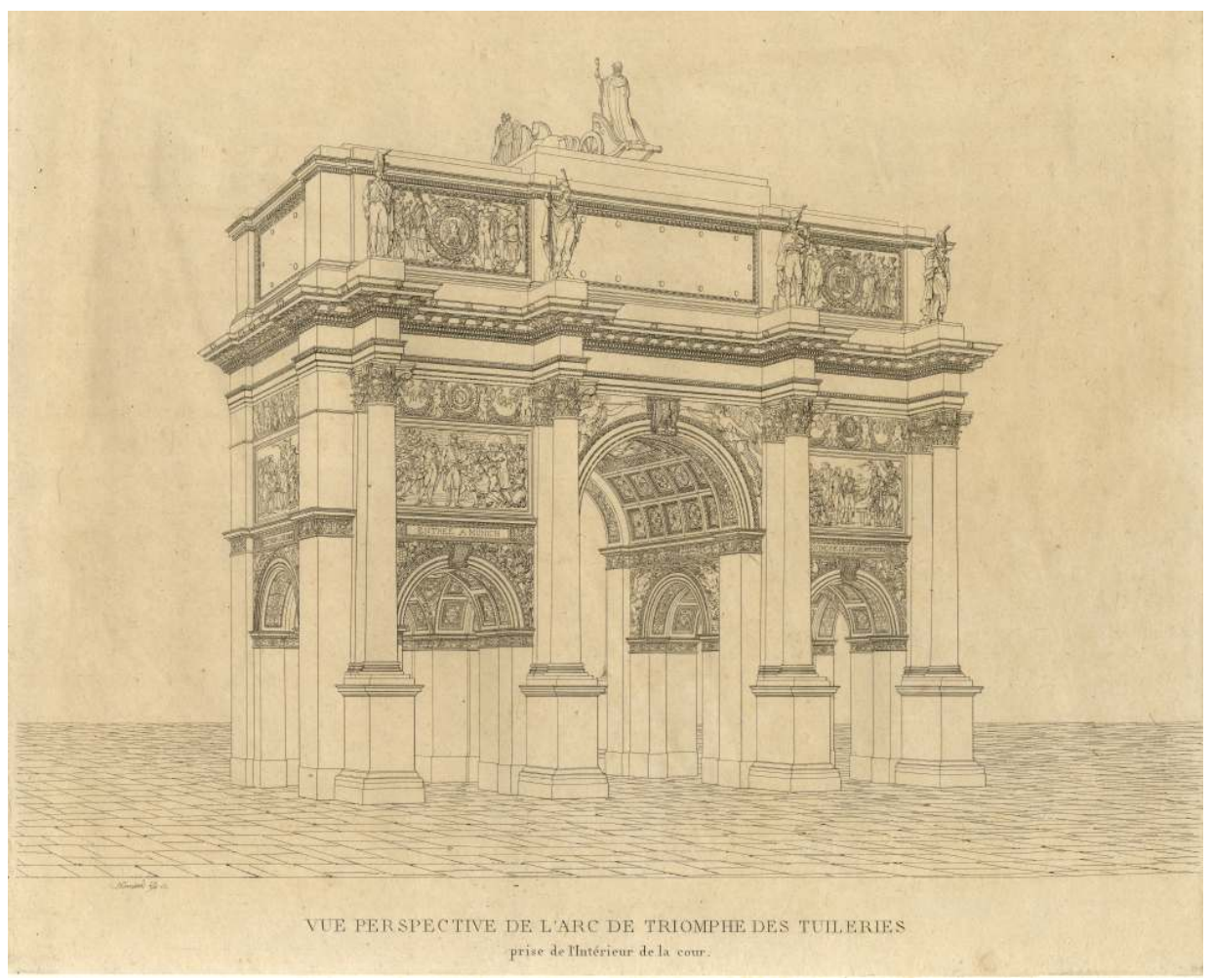

PLANCHE gRAVÉE, ARC DE TRIOMPHE DES TUILERIES, ÉRIgÉ EN 1806 d'APRÈs LES DESSINS ET SOUS LA DiReCtion DE MM. C. PERCIER ET P.-F.-L. FONTAINE... PARIS, NORMAND ET fils, [VERS 1826]

CL. FONKENELL

2 Le monument fut édifié avec une grande diversité de matériaux, sans doute dans l'intention de lui conférer plus de splendeur et de richesse: la pierre de taille dialogue avec les marbres de couleur et le marbre blanc statuaire; la dorure vient rehausser certaines sculptures en bronze ou en plomb, faisant de l'arc du Carrousel un véritable répertoire des matières premières en usage dans la construction.

L'édifice, dont l'histoire est très bien documentée par plusieurs fonds d'archives ${ }^{1}$, présente la particularité d'avoir subi très rapidement d'importantes transformations. Certaines d'entre elles étaient justifiées par les bouleversements politiques et les changements de régime qui ont marqué toute la première moitié du siècle. D'autres s'expliquent par la fragilité de l'édifice. Ces transformations sont l'occasion de comparer, à différents intervalles, la manière dont sont résolus des problèmes et des besoins analogues. Après un rapide rappel des grandes campagnes de travaux et de restauration de l'arc, l'analyse de l'approvisionnement matériau par matériau, campagne après campagne, permet de dégager les constantes et les évolutions durant le XIX ${ }^{\mathrm{e}}$ et le début $\mathrm{du} \mathrm{XX}^{\mathrm{e}}$ siècle.

\section{L'Arc de triomphe : une histoire mouvementée}

4 La construction de l'arc de triomphe fut décidée et menée à bien dans un temps très court. L'empereur Napoléon $I^{\text {er }}$ en commanda le projet à l'architecte Pierre-FrançoisLéonard Fontaine le 13 février $1806^{2}$. Le 26 février, un décret impérial précisait le mode de 
financement et les délais impartis pour sa réalisation ${ }^{3}$. Les fondations furent jetées au mois d'avril et la première pierre fut posée symboliquement le 7 juillet. Les colonnes de la face est étaient posées le 20 novembre suivant et le gros œuvre de l'arc était terminé le 26 avril 1807, mais l'empereur, toujours en campagne, ne le vit que le 4 janvier $1808^{4}$.

La décoration sculptée fut élaborée par Dominique-Vivant Denon, directeur du musée Napoléon, entre juin et juillet $1806^{5}$ et son exécution fut confiée à une équipe d'une quinzaine de sculpteurs, assistés par plusieurs artisans d'art. Elle devait glorifier l'empereur et son armée, en évoquant les grands moments de la campagne militaire de 1805 (sur des bas reliefs), les grands corps militaires (représentés par des soldats placés au niveau de l'attique) et l'empereur lui-même, sur un char triomphal pour lequel on utiliserait les chevaux de Saint-Marc, prise de guerre de la République française en $1797^{6}$. L'exécution fut achevée au mois d'août 1808; seule la statue de l'empereur ne fut pas placée au sommet de l'arc'.

6 L'arc du Carrousel, monument politique par excellence, faillit ne pas survivre aux bouleversements de $1815^{8}$. Il en sortit en tout cas très mutilé : les chevaux de Saint-Marc regagnèrent Venise en septembre. Leur dépose entraîna la destruction du char et détériora la Victoire et la Paix, les deux allégories qui l'entouraient et tenaient les chevaux par la bride ${ }^{9}$. Les bas-reliefs de la campagne de 1805 furent déposés et leurs emplacements restèrent vides pendant quinze ans ${ }^{10}$.

7 Dès 1823, l'administration royale projeta de substituer aux victoires de Napoléon une évocation de la campagne d'Espagne et commanda six nouveaux bas reliefs sur ce sujet ${ }^{11}$, mais les sculptures furent entreprises si lentement que le travail n'était pas terminé au moment de la chute du régime en $1830^{12}$. Seul le groupe sommital fut restitué entre 1826 et 1828 en conservant, après quelques hésitations, les deux statues de plomb et en les complétant par quatre chevaux neufs, tirant le char de la Restauration ou de la Paix ${ }^{13}$. L'arc fut maintenu après les journées de Juillet, moyennant quelques discrètes mutilations (le sommet du sceptre orné d'un Louis XVIII en majesté fut enlevée ${ }^{14}$ ) ; les bas-reliefs de l'ère napoléonienne furent remis en place. Le programme sculpté est aujourd'hui dans l'état où l'a laissé la monarchie de Louis-Philippe.

8 L'arc du Carrousel exigea assez rapidement une campagne de restauration, qui fut longtemps différée : demandée dès 1888 par l'architecte Edmond Guillaume, elle donna lieu à des débats sur les techniques à employer et fut repoussée ${ }^{15}$. Alors que plusieurs parties saillantes se détachèrent en 1907, $1909^{16}$ et en 1912, c'est seulement entre 1930 et 1933 que l'architecte du palais, Albert Ferran, entreprit une grande restauration, sur des principes qui avaient été établis par son prédécesseur, Camille Lefèvre ${ }^{17}$.

9 Les financements de chacune de ces grandes campagnes de construction puis de restauration furent très hétérogènes. Sous le Premier Empire, le maître d'ouvrage exigea des délais très courts, mais n'hésita pas sur les crédits à débloquer pour les tenir. Le financement de la construction fut assuré par un crédit d'un million de francs à prélever sur la caisse de la Grande Armée par décret du 26 février 1806. Il fut complété par un autre crédit d'un million pour le programme sculpté, décidé le 13 mai suivant ${ }^{18}$. Les travaux s'élevèrent en réalité à 1400000 francs environ ${ }^{19}$.

10 Les périodes qui suivirent furent marquées en revanche par des crédits beaucoup plus parcimonieux et par un allongement de la durée du chantier. Sous la Restauration, le comte de Forbin dut négocier avec son administration de tutelle pour obtenir de rétribuer 
de manière correcte les sculpteurs des bas-reliefs en marbre. Il est possible que les prix proposés, trop bas, aient dissuadé les premiers artistes pressentis pour les réaliser ${ }^{20}$.

11 Enfin, la restauration de 1930 s'effectua dans un contexte financier difficile et il fallut deux importants mécènes pour la rendre possible. Un Américain, Thomas Cochran, donna 500000 francs qui constituèrent le financement principal du chantier ${ }^{21}$. Ce premier don fut complété par un reliquat du legs fait en 1926 par Paul Marmottan, grand admirateur du Premier Empire et fondateur du musée et de la bibliothèque qui portent son nom ${ }^{22}$. L'administration des Beaux-Arts compléta sur son budget les fonds obtenus par ces deux mécénats ${ }^{23}$. Aux difficultés financières s'ajoutèrent les effets de la crise de 1929, entraînant une hausse des prix galopante. Le chiffrage final du dernier devis de maçonnerie ${ }^{24}$ fut augmenté de $25 \%$ pour «tenir compte d'une majoration pour application des assurances sociales, augmentation des matériaux et main d'œuvre au moment de l'exécution, c'est-à-dire au $2^{e}$ trimestre $1930 »$ ! Toujours pour raisons d'économie, on fit le choix de découper le chantier en deux tranches : la première portant sur les faces sud et ouest, la seconde sur les faces nord et ouest. On limitait ainsi les frais d'échafaudage, mais on doublait le temps de chantier qui s'échelonna au total sur plus de deux ans. Cette organisation obligea à des devis rectificatifs de maçonnerie pour la seconde tranche ${ }^{25}$.

L'arc du Carrousel, de sa construction (1806-1808) à sa transformation (1824-1830) et à sa restauration (1930-1933), fut donc géré de manières très différentes. Plusieurs régimes successifs et plusieurs administrations, avec des principes et des moyens différents, durent prendre en charge cet édifice dont la portée politique était manifeste. Si ces transformations expliquent en partie les changements d'approvisionnement en matériau, elles n'en sont pas l'unique raison, comme le montre une étude matériau par matériau.

\section{L'approvisionnement du chantier en pierre}

\section{La pierre à bâtir et le marbre de l'ordre corinthien}

13 Le mémoire général des travaux de maçonnerie ${ }^{26}$ montre que les pierres utilisées par Fontaine pour construire l'arc du Carrousel proviennent d'île-de-France et l'analyse des blocs en place aujourd'hui permet de préciser leur provenance ${ }^{27}$. Les assises inférieures sont en liais qui, d'après l'analyse, provient du sud de Paris : de Montrouge ou d'Arcueil. Les parties au niveau des arcs sont en pierre de Conflans et le reste en pierre de l'IsleAdam. Si les deux premiers gisements sont bien connus et exploités au moment de la construction de l'arc, Fontaine fit confiance à des fronts de carrière nouvellement ouverts à L'Isle-Adam, qui devaient, à l'épreuve, se révéler déficients. Dès 1828, certaines parties de l'attique de l'arc, où cette pierre avait été employée, durent être refaites ${ }^{28}$. Un mémoire qui semble avoir servi pour l'arc du Carrousel témoigne de l'état du savoir sur les carrières du bassin parisien au début du $\mathrm{XIX}^{\mathrm{e}}$ siècle $^{29}$. Le texte sur lequel s'appuie ce mémoire n'est autre que l'enquête réalisée en 1678 par l'Académie royale d'architecture à la demande de Colbert, mais le rédacteur a entrepris de découper le procès-verbal d'origine en deux colonnes pour distinguer ce qui relève de l'analyse des monuments parisiens d'une part et de la visite des carrières de l'autre. Il a par ailleurs intégré au fil de son découpage des notes inédites sur les pierres employées pour la restauration de la basilique Saint-Denis et de l'arc de l'Étoile, mélangeant les données anciennes et les 
informations les plus récentes et témoignant ainsi de la continuité des pratiques entre l'Ancien Régime et le Premier Empire.

L'architecte connut plusieurs déboires avec les colonnes. Il aurait souhaité utiliser du granit ${ }^{30}$, mais il dut y renoncer en raison des délais et tirer du magasin des marbres de Chaillot des colonnes de marbre du Languedoc qui avaient été taillées pour le Grand Trianon, mais n'avaient pas été employées ${ }^{31}$. L'analyse confirme que les marbres proviennent des carrières de Caunes-Minervois, dans le département de l'Aude, exploitées du temps de Louis XIV. Lors de la pose, un accident survint à la deuxième colonne de gauche de la face est : «Les liens et les cales qui l'enveloppaient ont glissé au moment où elle s'est trouvée entièrement suspendue, et le sommet au dessous de l'astragale s'est rompu, dans l'un des fils terrasseux dont le marbre de Languedoc est ordinairement rempli ». Une réparation "par des mastics à feu et rassemblé[e] par de forts goujons en fer » fut nécessaire ${ }^{32}$, l'architecte assurant que la solidité n'en serait pas affectée. Si l'avenir semble lui avoir donné raison, l'entablement de l'ordre principal, en revanche, présente aujourd'hui d'importants désordres, liés là aussi à l'emploi d'un matériau de récupération. Sa frise est constituée de panneaux de marbre rouge proche de la griotte, mais dont la provenance n'est pas indiquée par les archives. Les morceaux qui recouvrent la face des ressauts à l'aplomb des colonnes ont dû être liaisonnés avec le reste de la frise par des gougeons de fer. En rouillant, ils ont provoqué l'éclatement du marbre, voire l'écartement sensible des blocs. L'emploi de matériaux de récupération n'allait pas sans impact sur la longévité et la stabilité de l'édifice et explique les campagnes de restauration dont il fit l'objet quatre-vingt ans après son achèvement.

L'emploi de matériaux de récupération devait également avoir une influence sur l'architecture de l'arc. Dans son Journal, Fontaine affirme qu'il détermina les dimensions de l'édifice en fonction de son contexte urbain et palatial: par sa taille, il devait s'accorder au palais des Tuileries ${ }^{33}$. L'architecte dit-il la vérité, ou s'agit-il d'un vœu pieu ? Dans l'architecture classique, un système de proportion est censé lier toutes les parties de la composition entre elles ; il est généralement calculé à partir du diamètre de la colonne. Cette dimension de base, Fontaine ne pouvait pas la définir librement puisque il utilisa des fûts de colonnes déjà existants. La nécessité d'insérer des colonnes allogènes dans son projet explique peut-être certaines différences entre les proportions de l'arc du Carrousel et ses modèles directs : l'arc de Septime Sévère et surtout celui de Constantin (ill. 2). 
III. 2 : Comparaison entre les proportions de l'arc du Carrousel (à droite) et l'arc de Constantin (à gauche)

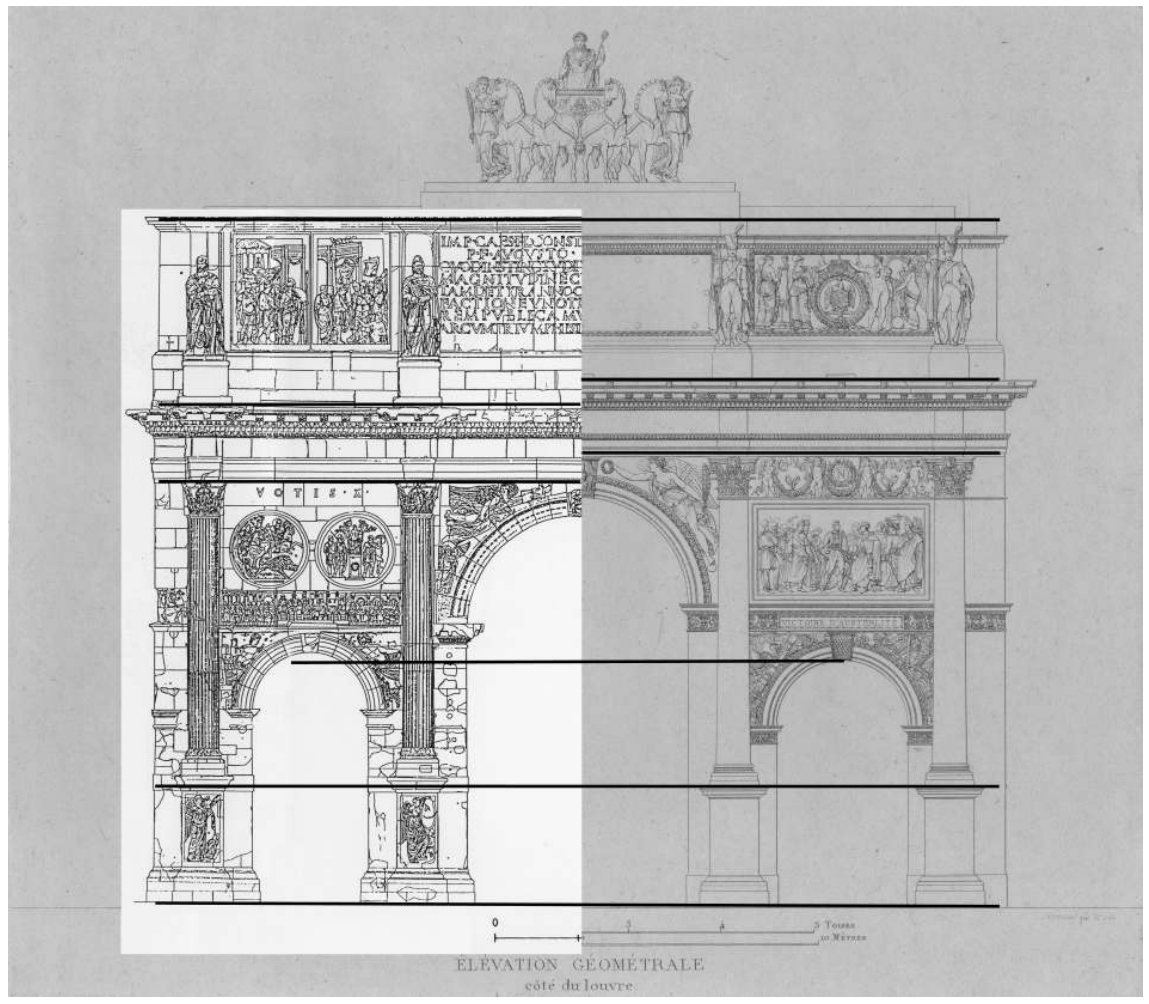

Dessin de l'auteur à partir de Arc de triomphe des Tuileries... Normand et fils et le relevé de Pensabene, Patrizio et Panella, Clementina, Arco di Costantino tra archeologia et archeometria. Rome, L'Erma di Bretschneider, 1999

Pour placer ses colonnes, Fontaine a dû surélever l'entablement et réduire ainsi la hauteur de l'attique, qui présente une élévation très inférieure à celle des précédents antiques dont il s'inspire.

À l'occasion du réaménagement des jardins du Carrousel en 1888, l'architecte Guillaume dut mener une campagne de réparation sur l'arc, dont plusieurs parties de corniche furent refaites. Cette campagne fut perturbée par un débat entre l'architecte et l'administration des monuments historiques sur les techniques de restauration, débat largement relayé par la presse durant le mois de mars $1889^{34}$. Pour des raisons de rapidité, Guillaume avait renoncé à remplacer les pierres détériorées et avait proposé d'y substituer un "ciment métallique ». Ce type de ciment, mis au point autour de $1850^{35}$ et obtenu avec des scories métalliques (en général des oxydes de zinc) associées à des matières silicieuses et des colorants pouvait être employé en ragréage, au lieu de changer par une reprise en sous-œuvre des pierres rongées ${ }^{36}$, ou bien en traitement de surface pour consolider des parties détériorées : appliqué au pinceau, il « adhère parfaitement à la pierre, lui rend l'apparence d'une taille fraîche et forme à la surface une enveloppe protectrice contre les intempéries" selon une revue scientifique de l'époque ${ }^{37}$. La commission des monuments historiques s'inquiéta de la stabilité du matériau dans le temps, et en particulier de l'évolution de sa couleur qui risquait de faire ressortir les parties restaurées ${ }^{38}$. Charles Garnier ${ }^{39}$, consulté à ce propos, s'opposa à son emploi pour des raisons de déontologie de la restauration: un "monument artistique " doit être maintenu en état et il faut arrêter les causes de dégradation, sans chercher à lui rendre 
une nouvelle jeunesse. Par ailleurs, les surfaces à réparer lui paraissaient trop importantes pour utiliser du ciment métallique. Le débat sur l'emploi de ce matériau semble avoir empêché Guillaume de mener à bien sa restauration; le jardin devait en effet ouvrir au mois de juin 1889 et il est peu probable que l'architecte ait eu le temps de commander la pierre finalement préconisée. En 1912, l'architecte Victor Blavette, qui projeta à nouveau une restauration de l'arc, se prononça à son tour en faveur de la pierre ${ }^{40}$.

Durant la restauration de 1930, l'emploi du ciment métallique ne fut jamais envisagé. En revanche, l'architecte Lefèvre hésita sur le choix de la pierre, et produisit trois devis sur ce sujet entre la fin de 1929 et le début de $1930^{41}$. Il se décida finalement pour la pierre de Chauvigny, extraite des environs de Poitiers (à $310 \mathrm{~km}$ de Paris) et présentant une structure oolithique en petits grains. Aujourd'hui encore, cette pierre au faciès particulier se reconnaît aisément sur les parements de l'arc (ill. 3).

\section{3 : Insertion de la pierre de Chauvigny (C) dans les parements en pierre du bassin parisien de} Percier et Fontaine

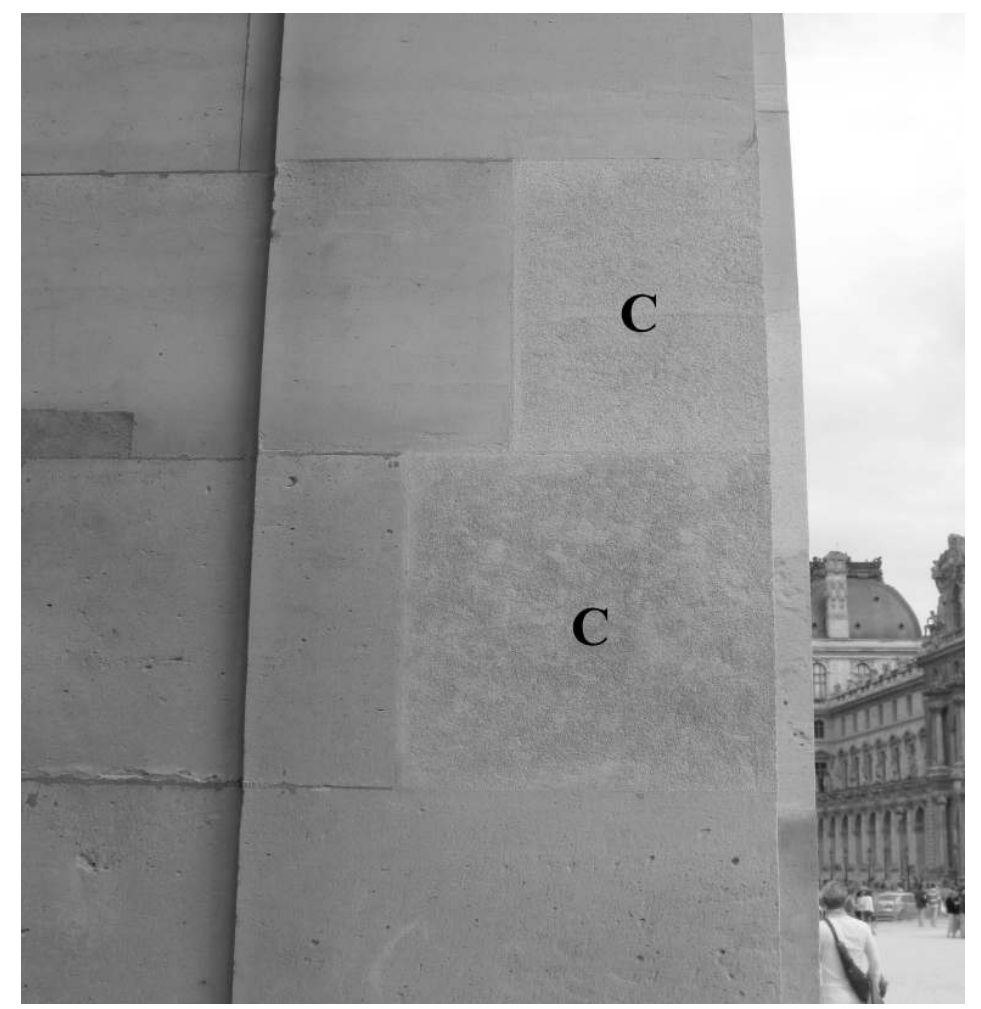

Cl. Fonkenell, 2008

19 Le choix de ce matériau, utilisé à la même période sur de grands chantiers modernes comme le placage des façades de la faculté de Médecine de Paris, permettait une économie de 202 107,53 francs, soit presque un tiers du montant total du devis ${ }^{42}$.

\section{Le marbre statuaire}

20 Le programme décoratif de l'arc de triomphe du Carrousel prévoyait un ensemble important de sculptures en marbre: les six bas-reliefs de grande taille illustrant la campagne militaire de 1805 mesuraient 1,75 m de haut sur 3,5 $\mathrm{m}$ de long environ, tandis 
que les soldats de la Grande Armée étaient représentés à l'attique plus grands que nature (3,8 $\mathrm{m}$ de haut en moyenne). Pour réaliser les rondes-bosses, il fallait disposer de blocs monolithes, tandis que les grands bas-reliefs étaient sculptés dans trois ou quatre morceaux, dont les joints étaient dissimulés dans les motifs de la composition.

Le marbre blanc statuaire, «cette matière infiniment précieuse par la difficulté de se la procurer ${ }^{43}$ " provenait des carrières de Carrare. Il n'avait pas été extrait pour l'occasion, puisque les ordres furent donnés pour son transport avant que le programme sculpté ne soit arrêté et seule une partie des quatre-vingt-deux blocs transportés à Paris devait être utilisée pour l'arc. Denon en demanda un état pour déterminer ceux qui pouvaient servir à l'arc du Carrousel ${ }^{44}$ et s'enquit également auprès de Chinard de toutes les informations possibles sur ces blocs ${ }^{45}$. Le directeur du musée Napoléon plaidait également pour que l'on reconstitue à Paris un important dépôt de marbres, les réserves constituées par Louis XIV étant arrivées à épuisement. Il pensait qu'un stock de cent cinquante à deux cents blocs de marbre statuaire et autant de marbre coloré était nécessaire pour faciliter les travaux à faire dans les maisons impériales et au musée ${ }^{46}$. Le marbre pour l'arc de triomphe fut transporté en plusieurs chargements qui passaient par Marseille et $\mathrm{Arles}^{47}$. La fin du parcours s'effectuait uniquement par voie d'eau ${ }^{48}$ probablement par le canal du Centre (ou canal du Charolais) qui reliait la Saône et la Loire et qui avait été achevé en $1793^{49}$. Le marbre pouvait ensuite être transporté toujours par bateau en passant du bassin ligérien à la Seine par le canal de Briare. L'ensemble du transport était assuré par le ministère de l'Intérieur qui semble l'avoir prit en charge budgétairement ${ }^{50}$; il était facilité par le fait que Carrare faisait partie de la principauté de Lucques, un état sous dépendance française, gouverné par la princesse Elisa Bonaparte. Le transport de Carrare à Paris prit dix mois environ: ordonnés le 10 mai $1806^{51}$, les blocs arrivèrent dans la capitale le 18 mars $1807^{52}$. Ils furent stockés au magasin de Chaillot, à l'exception des pierres destinées pour le Carrousel, déchargées sur le port Saint-Nicolas et réparties entre les sculpteurs.

Sous la Restauration, le nouveau programme sculpté exigea des blocs de marbre, mais leur provenance fut différente. Ils furent fournis en juin 1829 par la carrière de SaintBéat, gérée par Layerle Capel, fournisseur habituel de l'administration des beaux-arts. Les sculpteurs les retirèrent en magasin principalement en octobre 1829. La forme des blocs avait été déterminée en fonction de la composition de chacun des bas-reliefs (ill. 4) ${ }^{53}$. 
III. 4 : Schéma pour la découpe des marbres (à gauche), à partir des dimensions des blocs disponibles en carrière (à droite)

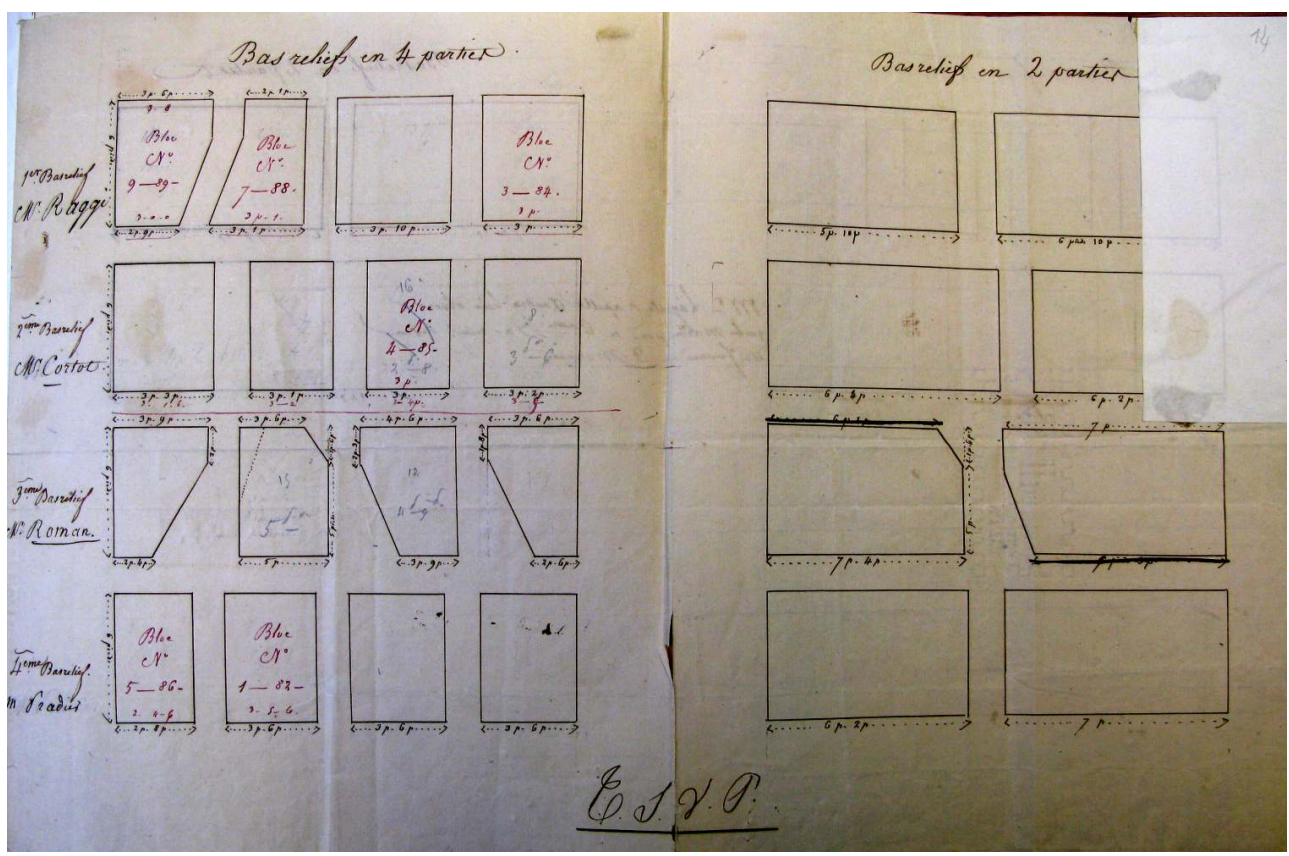

Document annexé au registre d'entrée et de sortie des blocs, vers 1827, Arch. des musées nat., 8 DD 7 I Cl. Fonkenell

Quelles sont les raisons de ce changement d'approvisionnement? Les évolutions politiques après la chute de l'Empire ont sans doute rendu le marbre italien plus difficile d'accès et plus cher. Par ailleurs, l'administration cherchait à promouvoir des marbres français. Le comte de Clarac, conservateur des sculptures du musée du Louvre, compare le Saint-Béat au marbre de Paros pour la « contexture » et l'estime supérieur au Carrare par son homogénéité qui ne comprend presque aucune veine; il loue enfin son blanc exceptionnel. L'ingénieur et scientifique Louis-Étienne Héricart de Thury, inspecteur général des carrières, va également dans ce sens dans son rapport sur les carrières françaises. Il n'est pas sûr que les sculpteurs aient entièrement partagé leur avis ; en tout cas, l'utilisation de ce type de marbre ne connut qu'un développement limité54.

\section{L'approvisionnement du chantier en métal}

\section{Le plomb (1806-1808)}

La réalisation du groupe sommital ${ }^{55}$ qui utilisait les quatre chevaux de Saint-Marc, devait être en métal, pour s'accorder avec les sculptures remployées. Denon proposa, pour des raisons d'économie, d'utiliser du plomb doré, dont le montant était évalué à 104000 francs contre 244000 francs pour un groupe en bronze sans dorure ${ }^{56}$. Il le justifiait par le précédent de la statue de Louis XIV sur la place des Victoires, information erronée puisque les groupes pour la place, d'abord projetés en plomb avaient finalement été fondus en bronze $\mathrm{e}^{57}$. Le sculpteur François-Frédéric Lemot fut chargé du modèle et sans doute de l'exécution. Les quantités de plomb employées pour la fonte montèrent au total à 16,4 tonnes et se répartissent approximativement en $4,03 \mathrm{t}$. pour la statue de l'empereur, 5,02 et 5,28 t. pour chacune des deux allégories féminines et $2,24 \mathrm{t}$. pour le 
char. Une perte de $10 \%$ de matière était prévue au cours des opérations de fonte et de réparage ${ }^{58}$. L'économie réalisée était estimée à 140000 francs, soit plus de la moitié du coût d'un groupe en bronze ${ }^{59}$ et devait être d'autant plus grande que le plomb utilisé ne fit l'objet d'aucune sélection particulière, en terme de qualité de minerai, de provenance ou de pureté ; il était composé à partir d'éléments hétérogènes issus de récupérations faites dans les magasins, et même sur les anciens scellements qui avaient servi aux chevaux de Venise sur la grille des Tuileries ${ }^{60}$.

L'utilisation du bronze fut réduite au minimum. Le char présentait une structure particulière : il était « en plomb doré, recouvert extérieurement et intérieurement de feuilles de laiton et de sculptures en bronze ${ }^{61}$ ». Le bronze seul ne fut utilisé que pour « les traits des chevaux, les harnais, la flèche du char, le sceptre etc... qui ne peuvent être exécutés qu'en cette matière ${ }^{62}$ » et pour les chapiteaux et les bases des colonnes. Les archives ne livrent d'indications ni sur sa provenance ni sur la nature de sa composition.

\section{Le bronze (1826-1828)}

La fonte de 1828, dont le modèle fut réalise par le baron François-Joseph Bosio et l'exécution par Charles Crozatier, est très bien connue (ill. 5) ${ }^{63}$.

III. 5 : Le groupe de couronnement de l'arc, comprenant les renommées en plomb de doré de François-Frédéric Lemot (1806-1808), et le char de la Restauration attelé de quatre chevaux en bronze de François-Joseph Bosio (1826-1828)

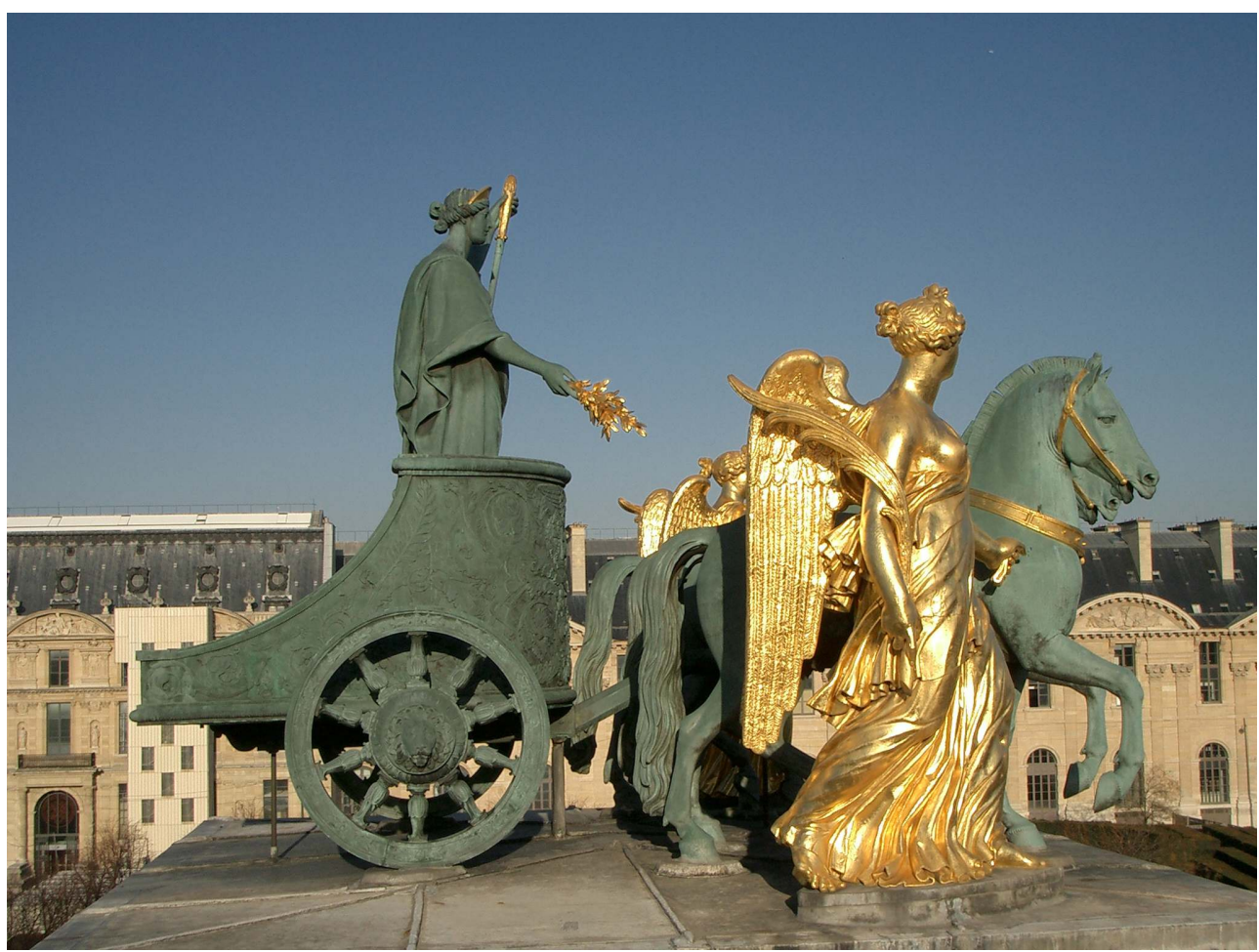

Cl. François Drocourt, 2003

Le matériau utilisé était un bronze de récupération, provenant du déclassement de canons étrangers que possédait le ministère de la Guerre. Le comte de Forbin, directeur des Musées royaux souhaita s'adjoindre un expert scientifique, connu par une lettre autographe sans date signée d'Arcet ${ }^{64}$. Cet expert doit être identifié avec Jean-Pierre- 
Joseph d'Arcet, chimiste spécialisé dans les alliages métalliques. Il avait déjà été consulté dans plusieurs opérations analogues, notamment pour la fonte de la colonne Vendôme ou la fonte de la statue d'Henri IV. Il est par ailleurs l'auteur d'un Mémoire sur l'art de dorer le bronze, publié en $1818^{65}$, et d'une méthode pour titrer l'argent ${ }^{66}$. Une commission fut nommée pour suivre et statuer sur les phases successives de la fonte. Seul le procèsverbal de la première réunion en date du 9 juillet 1827 est conservé. La commission était composée du comte de Forbin, directeur des Musées royaux, d'Alexandre de Cailleux, secrétaire des Musées royaux et directeur de l'École des beaux-arts, du baron LouisJacques Thénard, chimiste et homme de sciences et du comte de Clarac ${ }^{67}$. Thénard procéda à l'analyse de la qualité des différents bronzes, sans doute au Collège de France, à partir du 18 juillet suivant. Trente-quatre canons furent analysés: quatorze pièces allemandes, une pièce prussienne, une pièce de la Confédération du Rhin, une pièce hollandaise, dix pièces anglaises, une pièce italienne et six pièces espagnoles. Quoique le protocole d'analyse ne soit pas décrit, il est partiellement reconstituable à partir du rapport fourni sur la composition de ces bronzes : un échantillon fut prélevé sur chaque pièce et porté à la fusion. Le point de fusion ne se situant pas à la même température suivant les composants ${ }^{68}$, il fut possible de les séparer par échauffement progressif ${ }^{69}$. Une seconde opération identifia la part d'oxyde de chacun des trois métaux principaux : le plomb, le cuivre et l'étain. Ces analyses permirent de classer les bronzes en trois catégories et de rejeter deux canons, contenant trop de plomb. Les canons furent répartis en plusieurs groupes pour assurer la plus grande homogénéité possible aux fontes prévues par Crozatier.

28 Le poids des nouvelles statues est connu par une note du fondeur: les quatre chevaux exigèrent 9,44 tonnes de bronze, le char 4,89 tonnes et la statue 3,956 tonnes $^{70}$. Les canons fondus pesaient au total 16,503 tonnes $^{71}$. La technique de mise en œuvre employée par Crozatier, une fonte au sable d'un seul jet, permit également d'importantes économies, en réduisant le nombre d'opérations par rapport à une fonte à la cire perdue et en évitant l'emploi d'une lourde armature métallique ${ }^{72}$. L'administration dressa à ce sujet un tableau de comparaison entre le prix de la fonte du Carrousel, évalué à 114475 francs, et celle de la statue équestre d'Henri IV sur le Pont Neuf, réalisée en $1817^{73}$, estimé à 385870 francs. Si la comparaison entre les deux opérations est sans doute discutable, en particulier parce que certaines dépenses identiques d'un chantier à l'autre furent parfois prises en charge par l'administration et parfois par l'entrepreneur, ce mémoire témoigne bien de la volonté d'économie et d'efficacité de l'administration royale, toujours désireuse d'améliorer et de rentabiliser ses procédures.

L'analyse de l'approvisionnement du chantier de l'arc du Carrousel sur une centaine d'années permet de dégager quelques remarques générales. $\mathrm{Au} \mathrm{XIX}^{\mathrm{e}}$ siècle, l'approvisionnement en pierre se transforme considérablement. Sous le Premier Empire, Fontaine procède selon une logique qui est encore celle de l'Ancien Régime : la pierre à bâtir provient du bassin parisien, de sources proches du chantier $(6 \mathrm{~km}$ pour la pierre d'Arcueil et de Montrouge), même si celles-ci tendent déjà à devenir plus lointaines depuis le XVIII ${ }^{e}$ siècle (23,5 km pour Conflans, $29 \mathrm{~km}$ pour l'Isle-Adam). Cent ans plus tard, à l'occasion de la restauration de Ferran, le chemin de fer permet d'aller chercher la pierre jusque dans le Poitou ( $210 \mathrm{~km}$ de Paris) avec le risque d'utiliser des faciès très différents du matériau d'origine. Le coût du transport n'est plus déterminant et c'est désormais la facilité d'extraction qui détermine le prix de la pierre, puisque la pierre lointaine de Chauvigny est bien moins chère que celle du Bassin parisien. 
XIX ${ }^{e}$ siècle, le matériau reste coûteux et explique que l'on continue, dans la lignée des siècles précédents, à pratiquer la récupération, comme on le voit chez Fontaine (les colonnes de marbre) et Denon (pour la fonte des sculptures en plomb), ou sous la Restauration, vingt ans plus tard, avec la grande opération de bronze de récupération provenant de pièces d'artillerie. Une évolution semble cependant s'esquisser pour le marbre: Denon voulait constituer un grand magasin de blocs prêt à l'emploi pour des projets à venir, selon une logique qui était celle de l'Ancien Régime. Sous la Restauration, des fournisseurs qualifiés assurent à la demande et en fonction de la sculpture à réaliser la fourniture de blocs adaptés.

31 Tous les matériaux sont cependant touchés par un même phénomène : le renouvellement constant de leurs caractéristiques qui rend leur mise en œuvre et leur vieillissement difficiles à évaluer. Fontaine subit le phénomène avec les nouveaux gisements de l'IsleAdam. À la fin du siècle, l'administration choisit de jouer la prudence sur un procédé nouveau, le ciment métallique coloré. Face à ces risques, on sollicite l'analyse scientifique pour mieux comprendre et prévoir le comportement des matériaux. Le conseil constitué autour du bronze de la fonte de 1828 en est un exemple remarquable.

\section{NOTES}

1. 1. La section « Histoire du Louvre" réalise actuellement conjointement avec Michel Goutal, architecte en chef du palais, une étude historique sur l'arc de triomphe, en vue d'établir un programme de restauration de l'édifice. Les dépouillements d'archives ont été effectués avec l'aide de Ronan Bouttier. Le présent article est issu de ces recherches. Cette étude est consultable au musée du Louvre, à la section «Histoire du Louvre» dépendant du département des Sculptures. Guillaume Fonkenell et Ronan Bouttier, L'Arc du Carrousel, étude historique, 2008. Je tiens par ailleurs à remercier Marie-Laure Ludot et François Drocourt pour leur aide constante et amicale.

2. 2. Les grandes dates de construction de l'arc sont notées dans Pierre-François-Léonard Fontaine, Journal, Paris, École nationale supérieure des beaux-arts, Institut français d'architecture, 1987, 1373 p. Voir t. I, p. 121-122, 128, 137-138, 145 et 150.

3. 3. Le Moniteur Universel, 5 mars 1806 , résumé du décret. Une copie complète se trouve dans Arch. des musées nat., N00, dossier « Arc du Carrousel ».

4. 4. Pierre-François-Léonard Fontaine, Journal, op. cit., t. I, p. 185.

5. 5. Marie-Anne Dupuy, Isabelle Le Masne de Chermont et Elaine Williamson éd., Vivant Denon, directeur des musées sous le Consulat et l'Empire: correspondance, 1802-1815, Paris, Éditions de la réunion des musées nationaux, 1999, 1468 p. Voir principalement lettres nº 927-3, AN67, 1492-2.

6. 6. Pour une présentation synthétique et illustrée du programme sculpté, voir Christophe Leribault, «La campagne d'Allemagne illustrée : l'arc de triomphe du Carrousel », Napoléon et le Louvre, Sylvain Laveissière dir., Paris, Fayard, 2004, p. 220-241.

7. 7. Isabelle Leroy-Jay Lemaistre, «Le Louvre et les Tuileries, deux palais dans la ville », Napoléon et le Louvre, Sylvain Laveissière dir., Paris, Fayard, 2004, p. 41-45. La date de la dépose de la statue de Napoléon, placée dans le char, fait l'objet d'un débat dont les données sont reprises et détaillées dans Guillaume Fonkenell et Ronan Bouttier, L'Arc du Carrousel, étude historique, op. cit. 
8. 8. Pierre-François-Léonard Fontaine, Journal, op. cit., t. I, p. 496.

9. 9. Marie-Anne Dupuy, Isabelle Le Masne de Chermont et Elaine Williamson éd., Vivant Denon, directeur des musées sous le Consulat et l'Empire : correspondance, 1802-1815, op. cit., lettre $n^{\circ} 3597-3$.

10. 10. Pierre-François-Léonard Fontaine, Journal, op. cit., t. I, p. 472.

11. 11. Arch. des musées nat., N00, dossier «Arc du Carrousel », mention dans une lettre du ministre de la maison du roi au comte de Forbin en date du 18 fév. 1824. Les marbres ébauchés par les sculpteurs et laissés inachevés sont conservés au musée du Louvre (sculptures, CC 256 à 258).

12. 12. Gérard Hubert, «L'art français au service de la Restauration, à propos des épaves de l'arc du Carrousel conservées au Louvre », Revue des arts, 1955, nº IV, p. 209-216.

13. 13. Arch. des musées nat., N00, dossier « Arc du Carrousel », années 1827 et 1828.

14. 14. L'extrémité du sceptre est conservée au département des Sculptures, ENT 1998-2.

15. 15. Arch. nat., $\mathrm{F}^{21} 6238$, lettre $\mathrm{n}^{\circ} 2231$.

16. 16. Arch. nat., $\mathrm{F}^{21} 6241$.

17. 17. Voir essentiellement Arch. nat., 64AJ 826, 832, 833 et 910.

18. 18. Arch. nat., $\mathrm{O}^{2} 840$, dossier « décoration et sculpture de l'arc de triomphe place du Carrousel».

19. 19. Un récapitulatif commode du financement de la construction se trouve dans le recueil comptable, Arch. nat., $\mathrm{O}^{2} 274$.

20. 20. Gérard Hubert, «L'art français au service de la Restauration, à propos des épaves de l'arc du Carrousel conservées au Louvre ", op. cit. et Arch. des musées nat., S6, article 3.

21. 21. Arch. nat., 64AJ 910, articles de presse de J. G. Lemoine dans L'écho de Paris et de Robert Laulan, tiré d'un journal non identifié.

22. 22. Lettre de l'architecte en chef du 10 juin 1937. Arch. nat., 64AJ 910.

23. 23. Rapport sans doute rédigé pour la presse. Arch. nat., 64AJ 910.

24. 24. Arch. nat., 64AJ 910.

25. 25. Arch. nat., 64AJ 910. Deux devis sont conservés, le premier portant sur les parties hautes, le second sur les parties basses.

26. 26. Arch. nat., $\mathrm{O}^{2} 314$, mémoire $\mathrm{n}^{\circ} 5$.

27. 27. Cette étude est menée par Lise Leroux du laboratoire de recherche des monuments historiques que je remercie pour ses indications.

28. 28. Pierre-François-Léonard Fontaine, Journal, op. cit., t. II, p. 772.

29. 29. Arch. des musées nat., N00, dossier «Arc du Carrousel». Pour l'enquête de 1678, voir Henry Lemonnier éd., Procès-verbaux de l'Académie d'architecture, Paris, Jean Schémi, Édouard Champion, 1911, t. I, p. 168 sq.

30. 30. Pierre-François-Léonard Fontaine, Journal, op. cit., t. I, p. 145 et Arch. nat., $\mathrm{O}^{2}$ 307, dossier « Arc du Carrousel », pièce 5.

31. 31. Pierre-François-Léonard Fontaine, Journal, ibid. L'hypothèse qui fait provenir ces colonnes du château de Meudon a été réfutée par Christophe Bourel Le Guilloux, dans Francesco Primaticcio Architetto, Sabine Frommel dir., Milan, Electa, 2005, p.231. Je n'ai pas encore effectué de vérification des mesures du Grand Trianon pour confirmer définitivement l'assertion de Fontaine 32. 32. Pierre-François-Léonard Fontaine, Journal, ibid.

33. 33. Pierre-François-Léonard Fontaine, Journal, op. cit., t. I, p. 126.

34. 34. Arch. nat., $\mathrm{F}^{21} 6238$.

35. 35. Pierre Larousse, Grand dictionnaire universel $d u$ XIX $X^{e}$ siècle, Paris, Administration du Grand dictionnaire universel, 1869, t. IV, p. 300, col. 4.

36. 36. Louis Figuier, L'année scientifique et industrielle, Paris, Librairie Hachette, 1887, $\mathrm{n}^{\circ} 31, \mathrm{p}$. 474-475. L'article précise que le ciment métallique a été employé pour restaurer la colonnade du Louvre, le Pont-Neuf, le conservatoire des arts et métiers et le Palais-Royal.

37. 37. Revue scientifique, t. XLIX, $\mathrm{n}^{\circ} 12$, p. 382. 
38. 38. Ibid., articles de La Petite presse et de L'Écho de Paris, 12 mars 1889.

39. 39. Arch. nat., $\mathrm{F}^{21} 6238$, lettre du 15 mars 1889.

40. 40. Arch. nat., $\mathrm{F}^{21}$ 6249, article de presse dans L'Éclair, 24 mai 1912 et lettre du 5 octobre suivant.

41. 41. Arch. nat., 64AJ 910.

42. 42. Second devis de 1929. Arch. nat., 64AJ 910.

43. 43. Marie-Anne Dupuy, Isabelle Le Masne de Chermont et Elaine Williamson éd., Vivant Denon, directeur des musées sous le Consulat et l'Empire : correspondance, 1802-1815, op. cit., lettre $n^{\circ} 1107$.

44. 44. Ibid., lettre $n^{\circ} 1024$.

45. 45. Ibid., lettre $n^{\circ} 1048$.

46. 46 . Ibid., lettre $n^{\circ} 986$.

47. 47. Arch. nat., $\mathrm{O}^{2} 840$, dossier «décoration et sculpture de l'arc de triomphe place du Carrousel».

48. 48. C'est ce que laisse entendre Dominique-Vivant Denon. Marie-Anne Dupuy, Isabelle Le Masne de Chermont et Elaine Williamson éd., Vivant Denon, directeur des musées sous le Consulat et l'Empire : correspondance, 1802-1815, op. cit., lettre $\mathrm{n}^{\circ} 1024$.

49. 49. Un canal, des canaux, catal. expos., Pierre Pinon dir., Paris, CMHS, 1986, p. 351.

50. 50. Aucune recherche n'a été effectuée dans ces fonds pour préciser éventuellement les informations fournies par la correspondance de Denon.

51. 51. La date de départ est mentionnée dans deux lettres: lettre de Denon au ministre de l'Intérieur du 2 août 1806 (Marie-Anne Dupuy, Isabelle Le Masne de Chermont et Elaine Williamson éd., Vivant Denon, directeur des musées sous le Consulat et l'Empire: correspondance, 1802-1815, op. cit., lettre $n^{\circ}$ 986) et lettre de Daru à Denon (Arch. nat., $\mathrm{O}^{2} 840$, dossier « décoration et sculpture de l'arc du Carrousel »).

52. 52. Marie-Anne Dupuy, Isabelle Le Masne de Chermont et Elaine Williamson éd., Vivant Denon, directeur des musées sous le Consulat et l'Empire : correspondance, 1802-1815, op. cit., lettre $n^{\circ} 1107$.

53. 53. Croquis des différents blocs de marbres, relié avec le registre d'entrée et de sortie des blocs. Voir en particulier la note à Layerle Capel sur le sixième bas-relief. Arch. des musées nat., 8 DD 7.

54. 54. Charles-Othon-Frédéric-Jean-Baptiste de Clarac, Musée de sculpture antique et moderne ou Description historique et graphique du Louvre et de toutes ses parties, Paris, Imprimerie royale, 1841, t. I, p. 707. Louis-Étienne Héricart de Thury, «Rapport fait à la Société d'encouragement pour l'industrie nationale sur l'état actuel des carrières de marbre de France ", Annales des mines, $\mathrm{n}^{\circ} 8$, 1823, p. 42. Je remercie G. Bresc-Bautier pour ses indications sur l'emploi du marbre de SaintBéat.

55. 55. L'étude de ce groupe est actuellement en cours par Annick Texier du laboratoire de recherche des monuments historiques.

56. 56. Marie-Anne Dupuy, Isabelle Le Masne de Chermont et Elaine Williamson éd., Vivant Denon, directeur des musées sous le Consulat et l'Empire : correspondance, 1802-1815, op. cit., lettre n 927-3.

57. 57. Geneviève Bresc, «Louis XIV, place des Victoires », Art ou politique, arcs, statues et colonnes de Paris, Béatrice de Andia dir. Paris, Action artistique de la ville de Paris, 2008, p. 64.

58. 58. Quittance du 19 oct. 1806. La conversion a été effectuée sur la base de la livre de Paris, à $489.5 \mathrm{~g}$.

59. 59. Aperçu des dépenses de l'arc, 22 juillet 1806. Arch. des musées nat., N00, dossier «Arc du Carrousel».

60. 60. Marie-Anne Dupuy, Isabelle Le Masne de Chermont et Elaine Williamson éd., Vivant Denon, directeur des musées sous le Consulat et l'Empire : correspondance, 1802-1815, op. cit., lettres $n^{\circ} 1368$ et n - 1409.

61. 61. Arch. des musées nat., N00, dossier « Arc du Carrousel». 
62. 62. Arch. nat., $\mathrm{O}^{2} 840$, dossier « décoration et sculpture de l'arc de triomphe place du Carrousel ».

63. 63. Rapport sur la fonte, 29 déc. 1929. Arch. des musées nat. N00, dossier « Arc du Carrousel». 64. 64. Arch. des musées nat., N00, dossier « Arc du Carrousel».

65. 65. Bibl. nat. de France, Imprimés, V 30514. D’Arcet continua ses recherches sur la dorure jusqu'à la fin de sa vie, comme en témoigne une Note sur les procédés de dorure par la voie humide de 1843 dont un exemplaire est consultable à la BnF, Imprimés, VP 5863.

66. 66. Mémoire sur la détermination des quantités de plomb nécessaires pour passer à la coupelle les essais d'argent à différents titres; un exemplaire est consultable à la Bibl. nat. de France, VP 13513.

67. 67. Arch. des musées nat., N00, dossier «Arc du Carrousel»; lettre du vicomte de la Rochefoucauld au comte de Forbin.

68. 68. L'étain entre en fusion à $505 \mathrm{~K}$, le plomb à $600 \mathrm{~K}$ et le cuivre à $1357 \mathrm{~K}$.

69. 69. Tableau récapitulatif de l'analyse des pièces de canon. Arch. des musées nat., N00, dossier «Arc du Carrousel» .

70. 70. Note de Crozatier, Arch. des musées nat., N00, dossier "Arc du Carrousel ».

71. 71. Ce chiffre est celui donné par la liste de «Pesée des Canons/ au domicile de Mr Crozatier » (Arch. des musées nat., N00, dossier «Arc du Carrousel »), duquel on a déduit le poids des deux pièces qui ne furent pas retenues pour la fonte en raison de la trop grande quantité de plomb qu'elles contenaient.

72. 72. Lettre de réclamation de Crozatier du 31 mai 1828. Arch. des musées nat., N00, dossier « Arc du Carrousel».

73. 73. «Comparaison des dépenses occasionnées par l'érection de la statue équestre d'Henri IV... », Arch. des musées nat., N00, dossier « Arc du Carrousel ».

\section{RÉSUMÉS}

L'arc de triomphe du Carrousel, monument éminemment politique voulu par l'Empereur Napoléon et conçu par l'architecte Fontaine et le directeur des musées Denon, connut les aléas inhérents à ce type de monument dans un pays traversés par des changements de régime récurrents. Que ce soit sa construction (1806-1808), sa transformation après le retour des Bourbons (1826-1828) ou sa restauration, plusieurs fois demandée et repoussée jusqu'au XX siècle (1930-1933), toutes les phases de son existence furent marquées par d'importantes contraintes de délais, d'économie et des volontés politiques fortes, parfois contradictoires. Ces facteurs eurent une influence qui est décisive mais qui n'explique pas tout sur l'approvisionnement en matériaux du chantier. L'étude de ces approvisionnements est d'autant plus intéressante que des hommes et des administrations différentes furent confrontés à peu de temps d'intervalle à des problèmes analogues ; l'arc du Carrousel permet de voir les permanences et les évolutions dans les solutions qu'ils utilisèrent. L'apport en pierre à bâtir, en marbre, en plomb et en bronze illustre l'évolution des sources d'approvisionnement, l'importance de la pratique de la récupération au XIX ${ }^{e}$ siècle et surtout une évolution constante des caractéristiques des matériaux qui impose le recours de plus en plus important à l'expertise scientifique pour tenter de mieux maîtriser et comprendre leur évolution et leur vieillissement.

The Carrousel triumphal arc, apolitical monument "par excellence", desired by the Emperor Napoleon and devised by the architect Fontaine and by Denon, director of the imperial museums, 
came to know all the hazards inherent in this kind of monument set in a country that experienced so many political changes in such a short period of time. During its construction (1806-1808), its transformation when the Bourbons came back to the throne (1826-1828) or its restoration, so many times required before it was eventually made in the $\mathrm{XX}^{\text {th }}$ century (1930-1933), the building had to undergo considerable constraints in term of time, finance and politics. Studying the supplying of building material is all the more interesting because different men and administrations had to resolve similar problems in a short period of time. We can therefore analyse the permanence and the evolutions in the means used to work out these questions. It illustrates the progress in the search for material supply, the importance of architectural works' re-use in the XIX ${ }^{\text {th }}$ century and it throws light on the fact that in order to understand the constant change in the nature of these materials and their ageing, scientific expertise needed more and more to be called in.

Der Arc de triomphe du Carrousel, nach dem Willen Kaiser Napoleons gebaut, von dem Architekten Fontaine und dem Direktor der Museen Denon konzipiert, folgte als höchst politisches Denkmal dem Lauf der wechselnden Geschichte in einem Lande, das von andauernden Regimeänderungen getroffen wurde. In allen Phasen seiner Existenz also, vom Bau (1806-1808) über die Veränderung nach der Rückkehr der Bourbonen (1826-1828) bis zur mehrmals verlangten und im 20. Jahrhundert verschobenen Restaurierung (1930-1933) unterlag das Gebäude beträchtlichen Fristund Wirtschaftsverpflichtungen, sowie starkem und manchmal widersprüchlichem politischen Willen. Diese Faktoren, die zwar einen entscheidenden Einfluss ausgeübt haben, klären aber nicht alle Fragen zur Materialbeschaffung der Baustelle. Die Untersuchung dieser Beschaffung erweist sich um so interessanter, als Männer und verschiedene Verwaltungen in kurzem Zeitabstand auf ähnliche Probleme stoßen und dass sich zugleich Beständigkeit und Erneuerung in den getroffenen Maßnahmen erkennen lassen. Die Anfuhr von Bausteinen, von Marmoren, von Blei und Bronze zeugt von der Entwicklung der Beschaffungsquellen und von der häufigen Praxis der Wiederverwendung von Materialien im 19.Jahrhundert. Es erweist sich auch, dass man sich infolge der stetigen Entwicklung der Eigenschaften der Materialien immer mehr an wissenschaftliche Experten wendet, um Zustand und Alterung des Baus zu überprüfen.

\section{AUTEUR}

\section{GUILLAUME FONKENELL}

Guillaume Fonkenell, né le 9 mai 1975, ancien élève de l'École normale supérieure, agrégé de Lettres classiques, architecte D.P.L.G., est chargé de la section « Histoire du Louvre ", rattachée au département des Sculptures du musée du Louvre. Chargé de cours à l'École nationale des Chartes et à l'École du Louvre. Il prépare actuellement une thèse de doctorat sous la direction de Claude Mignot à l'Université de Paris-IV sur le Louvre et les Tuileries au temps des Lumières (1746-1792). Il a écrit sur les écuries royales et impériales, et sur Jules Hardouin-Mansart. guillaume.fonkenell@louvre.fr 